2. Finnigan S, van Putten M. EEG in ischaemic stroke: quantitative EEG can uniquely inform (sub-)acute prognoses and clinical management. Clin Neurophysiol. 2013;124(1):10-19.

3. Finnigan S, Wong A, Read S. Defining abnormal slow EEG activity in acute ischaemic stroke: delta/alpha ratio as an optimal QEEG index. Clin Neurophysiol. 2016;127(2):1452-1459.

Prehosp. Disaster Med. 2019;34(Suppl. 1):s87-s88

doi:10.1017/S1049023X19001821

\section{Tracking Patients in an Earthquake Response: The Bad,} the Better, and the Best

Miss. Paula Grainger

Canterbury District Health Board, Christchurch, New Zealand

Introduction: Tracking patients through health systems is fundamental to coordinated care provision. However, it is an inconsistent element of emergency preparedness. This presentation presents findings of a study undertaken after the 2011 Christchurch Earthquake, and the resultant nationally implemented changes.

Aim: The intent was to investigate options to improve patient tracking in a mass casualty event. By looking at one scenario involving a mass casualty presentation with the central responder disabled by electricity loss, standards of practice were outlined and made scalable to meet the needs of various events.

Methods: Clinical and clerical staff involved in the event's patient tracking were interviewed. Data were analyzed using thematic analysis and reported using the structure, process, and outcomes framework. ${ }^{1}$

Results: Structures were material and human resources. Material resources were identification number systems, technological requirements, disaster-specific documents, minimum data for entry, digital/paper/hybrid registration systems, and digital-paper integration. Human resources were role allocation, and familiarity of plans, roles, processes, tools, and facilities. Process identified the activities to manage unidentified patients, triage, registration, and ongoing tracking processes. Outcomes were management of patient flow, patient-care provision, and patient-family reunification.

Initial implementation was local. Structures and processes were agreed upon, with varying response levels according to the incident scope, while staying as close to business as usual for familiarity. National implementation followed via a Ministry of Health working group involving different district health boards. The group developed a consensus on the minimum data to be entered and the process to merge patient identities of initially unidentified patients. Written tools were shared for standardization.

Discussion: With inter-agency and inter-organization emergency response, standardized processes and information are required. Collaboration prior to events can mitigate issues when an event occurs.

\section{References}

1. Donabedian A. (1988). "The quality of care: How can it be assessed?". JAMA 260(12):1743-1748.

Prehosp. Disaster Med. 2019;34(Suppl. 1):s88

doi:10.1017/S1049023X19001833
Use of a Novel Electronic Patient Care Record System at Mass Gathering Events by St. John Ambulance Victoria Ms. Crystal Gao ${ }^{1,2}$, Dr. Zheng Jie Lim ${ }^{2,3,4}$,

Mr. Brendan Freestone ${ }^{2,3}$, Ms. Kristy Austin ${ }^{2,3}$,

Mr. Rob McManus ${ }^{2}$

1. Faculty of Medicine, Nursing \& Health Sciences, Monash University, Clayton, Australia

2. Event Health Services, St John Ambulance Victoria, Mount Waverley, Australia

3. Clinical Services, St John Ambulance Victoria, Mount Waverley, Australia

4. Ballarat Health Services, Ballarat Central, Australia

Introduction: The growing number of mass gathering events (MGEs) in Victoria has seen an increase in demand for event health services and the need for real-time reporting of medical incidents at these events.

Aim: Since 2016, St. John Ambulance Victoria has introduced an electronic patient care record (ePCR) system with the aim of improving patient care and satisfaction. It appears that this ePCR system is the first of its kind to be trialed at MGEs by a volunteer organization.

Methods: A qualitative study was conducted to determine strengths and limitations of the ePCR system by compiling results of surveys and interviews and through anonymous feedback from volunteers and patrons (event organizers, patients). This study is ongoing.

Results: It was found that the use of ePCR:

1. Allowed for collection of relevant data to assist in future planning of MGEs

2. Aids the overall coordination of first aid delivery at MGEs faster relaying of patient information to event commanders reduction of paperwork

improved ability to locate first aid crews using GPS tracking

3. Received positive feedback from first aiders, event organizers, and patrons

4. Was deemed easy-to-use (4/5), acceptable (4.3/5), and helpful (4.1/5) by our members

Discussion: These experiences demonstrate that ePCR is wellreceived, easy to use, and leads to improved patient satisfaction and treatment outcomes at MGEs. Furthermore, the ability to collect and analyze real-time data such as GPS location tracking, incidence heat maps, and patient demographics facilitate future event planning and resource allocation at MGEs. It is acknowledged that this study is preliminary, and the trialed use of an ePCR system has been limited to metropolitan areas and MGEs with $<1$ million patrons. The intent is to continue this study and explore the use of ePCRs at larger MGEs and events in rural or regional areas.

Prehosp. Disaster Med. 2019;34(Suppl. 1):s88

doi:10.1017/S1049023X19001845

\section{Watching to Save Lives}

Mr. Raphael Herbst, Dr. Eli Jaffe

Magen David Adom, Tel Aviv, Israel

Introduction: As Israel's National Emergency Medical Services (EMS) provider, Magen David Adom (MDA) is 
constantly looking for ways to improve the response to mass casualty incidents (MCIs). Previous research has shown that in an MCI situation, the demand for resources is disproportionate to the available resources, thus creating a dilemma of how to triage and treat the patients, as well as how to best prioritize and treat the critical patients.

Aim: Smartwatches have become an integral part of society. MDA constantly looks for ways to integrate new technologies into their emergency response protocols. Smartwatches were used in this experiment to determine if in an MCI, relaying live information to the dispatch center would improve the time it takes for emergency crews to effectively treat and transport critical patients.

Methods: A drill and scenario were designed to simulate an MCI in which there were 3 severe, 2 moderate, and 5 lightly wounded patients. There were then different colored smartwatches placed on each victim. The watches transmitted real-time blood pressure, pulse, and oxygen saturation readings to the dispatch center. The live information was transferred directly to responding teams. A second drill was conducted using the same scenario, same number of patients, but without watches to examine the differences in response times.

Results: MDA found that the use of smartwatches directly improved the times (by 3.27 minutes) in which emergency teams were able to reach the most severely wounded patients and evacuate them to the hospital in a timely manner.

Discussion: Using smartwatches to transmit live information to the dispatch center allowed for effective treatment and transport of patients in an MCI. Use of such information allows the dispatch center to direct teams to provide accurate treatment to the patients according to their needs.

Prehosp. Disaster Med. 2019;34(Suppl. 1):s88-s89

doi:10.1017/S1049023X19001857 